\title{
Kernos
}

Revue internationale et pluridisciplinaire de religion grecque antique

5 | 1992

Varia

\section{Sur Les Bacchantes de Théocrite}

\section{Maria Teresa Molinos Tejada}

URL : http://journals.openedition.org/kernos/1053

DOI : 10.4000/kernos. 1053

ISSN : 2034-7871

\section{Éditeur}

Centre international d'étude de la religion grecque antique

\section{Édition imprimée}

Date de publication : 1 janvier 1992

ISSN : 0776-3824

\section{Référence électronique}

Maria Teresa Molinos Tejada, «Sur Les Bacchantes de Théocrite », Kernos [En ligne], 5 | 1992, mis en ligne le 19 avril 2011, consulté le 21 avril 2019. URL : http://journals.openedition.org/kernos/1053 ; DOI : 10.4000/kernos.1053 
Kernos, 5 (1992), p. 109-117.

\section{SUR LES BACCHANTES DE THÉOCRITE}

L'expérience religieuse sous-jacente du culte de Dionysos diffère essentiellement de celle que l'on trouve dans le culte du reste des Olympiens et offre des possibilités de communion, d'identification avec la divinité, tout à fait absentes de la religion homérique.

Faut-il seulement rappeler que $\tau \dot{\alpha}$ ôp $\gamma 1 \alpha$ n'étaient pas des "orgies" dans le sens moderne du mot, mais des actes de culte accomplis au cours d'un

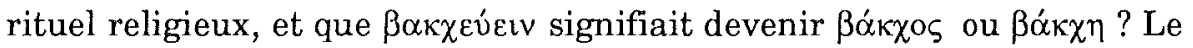
fidèle sentait que le dieu prenait possession de lui et pouvait, tant que l'extase durait, s'appeler du nom du dieu qu'il adorait. La musique, la danse, la ópeıß $\beta \alpha \sigma i \alpha . .$. l'aidaient à se libérer des inhibitions, des conditionnements que la raison et les règlements sociaux imposaient à sa conduite, l'aidaient à laisser la périphérie et à s'avancer dans la vraie nature des choses, la communion avec la divinité, l'extase.

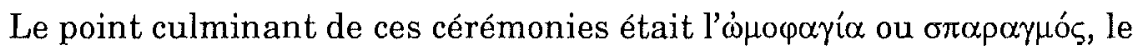
dépiècement et la manducation d'un animal fraîchement tué, encore plein de sang et de vie, victime dans laquelle on croyait voir la divinité. En absorbant son sang, on se sentait pénétré de la force du dieu.

Normalement il s'agissait d'un animal; le taureau était sans doute le plus représentatif. C'était une des formes les plus communes que prenait Dionysos pour se manifester à ses fidèles. Selon Plutarque ${ }^{1}$, les femmes d'Élis priaient le dieu de paraître en criant par deux fois $\alpha^{\prime} \xi_{\imath \varepsilon} \tau \alpha \hat{\rho} \rho \varepsilon$, et Dionysos avait aussi des prêtres appelés ßovkó $\lambda o l$; mais d'autres animaux étaient également censés représenter le dieu. Pouvait-il arriver que la victime fût un homme ? Quelques épithètes font penser à cette possibilité :

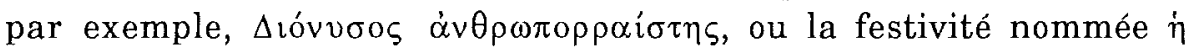
$\dot{\alpha} v \theta \rho \omega \pi \circ \theta v \sigma i \hat{\omega} v \beta \alpha \kappa \chi \varepsilon i \alpha ;$ le mythe même de Penthée pointe dans cette direction-là. Nous avons des renseignements épars ${ }^{2}$ sur des sacrifices

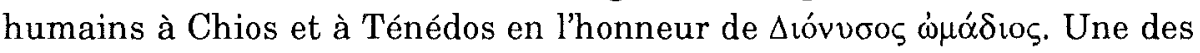
dénonciations entraînant la loi de bacchanalibus de 186 av. J.-C., qui prétendit régler le mouvement dionysiaque italien, était précisément le meurtre rituel... Cela fut possible. Les indications sont cependant très vagues et il y a certainement des érudits pour lesquels ces informations

1 Quaest. Gr., 36, 299b.

2 Cf. l'information recueillie par E.R. Dodds dans l'introduction à son édition des Bacchantes d'Euripide, p. XVIII-XX, et dans l'appendice I, Maenadism, in The Greeks and the Irrational, Berkeley, 1951, p. 270-282. 
n'ont pas de crédit. On a voulu voir aussi dans Les Bacchantes de Théocrite (v. 29) une indication de cette espèce. Il s'agirait d'une allusion voilée à la mort d'un jeune garçon; on a défendu la possibilité d'une excuse, même d'une apologie, d'un encouragement de la part du poète à commettre de tels faits.

Nous allons maintenant réviser ce vers, sans doute un des plus polémiques de l'œuvre de Théocrite, et à l'aide du Papyrus d'Antinoé, nous emprunterons une nouvelle voie de solution.

Les Bacchantes, comme les hymnes de Callimaque ou l'idylle des Dioscures, ne sont probablement pas de véritables hymnes religieux destinés à l'exécution du culte; il est beaucoup plus vraisemblable qu'ils soient, bien que remplis d'allusions et de formules rituelles, des compositions simplement littéraires écrites à l'occasion d'une fête, peut-être évoquées par elle.

La question de l'authenticité de l'idylle est un problème sur lequel les érudits ne se mettent pas d'accord. Wilamowitz soulignait les défauts formels, les gaucheries métriques et de construction, la maladresse de composition spécialement dans l'utilisation des particules ${ }^{3}$, mais le papyrus d'Antinoé ${ }^{4}$ et aussi le parchemin du Louvre ${ }^{5}$ pour la première partie du poème prouvent, qu'au moins au Ve siècle, Les Bacchantes étaient admises parmi les idylles authentiques de Théocrite. Aujourd'hui on est presque d'accord pour reconnaître au poète syracusain la paternité de l'idylle.

La narration du sacrifice de Penthée commence directement sans aucun préambule ni dédicace : Inô, Agavé et Autonoé, chacune à la tête

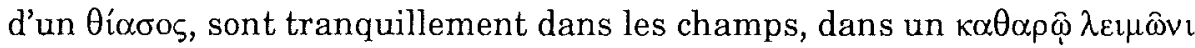
(v. 5) plus exactement, avec la connotation de pureté rituelle que к $\alpha \theta \alpha \rho \hat{\omega}$ possède, apprêtant des autels rustiques en l'honneur de Sémélè et de

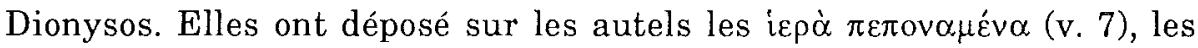

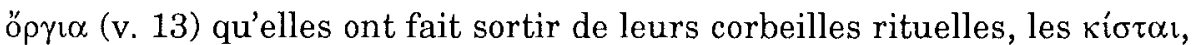

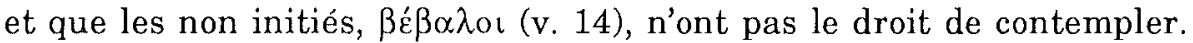
Elles se disposent donc, au contraire des bacchantes d'Euripide, à célébrer une cérémonie sacrée. Soudain elles aperçoivent Penthée qui, caché dans un arbre, guette la paisible scène. Prises d'une fureur subite, elles le

3 Die Textgeschichte der Griechischen Bukoliker, Berlin, 1906, Beilage 8, Lenai, p. $215 \mathrm{sq}$.

4 A.S. HunT-J. Johnson, Two Theocritus Papyri, London, 1930, p. 19-87 + Add. 207 in The Antinopolis Papyri III, London, 1967.

$5 \quad$ Perg. du Louvre 6678, publié par K. WEssely in WS, 8 (1886), p. 221-230, et Mitt. Pap. Erzh. Rainer, 2 (1888), p. 78 sq. Cf. aussi les corrections de lecture effectuées par J. BINGEN in CE, 57 (1982), p. 309-316. 
chassent et le mettent en pièces. La narration se déroule rapidement, sans s'attarder sur l'horrible spectacle avec des détails macabres.

Ensuite, avant le salut final, le $\chi \alpha$ ípor à Dionysos, à Sémélè et à ses sœurs, le poète tire de l'histoire du châtiment de Penthée une réflexion personnelle, une sorte de pieuse $\gamma \nu \omega \mu \eta$, habitude aussi ancienne qu'Hésiode (Erga, 270), que nous retrouvons souvent dans la littérature grecque. Ainsi le messager des Bacchantes d'Euripide, à la fin de son récit

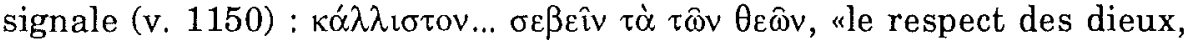
c'est ce qu'il y a de plus beau». Des réflexions similaires se retrouvent dans deux des Hymnes de Callimaque, poèmes qui ressemblent le plus à notre idylle. Dans l'hymne à Déméter, après la narration du châtiment du pauvre Érysichton, dévoré par une insatiable faim, le poète s'écrie (VI, 116 sq.) : «Déméter, qu'il ne me soit point ami celui que tu n'aimes pas... mauvais voisins pour moi tes ennemis... Filles chantez, $\Delta \alpha ́ \mu \alpha \tau \varepsilon \rho \mu \varepsilon_{\varepsilon} \gamma \alpha$ $\chi \alpha \hat{\imath} p \varepsilon »$.

Dans le troisième hymne, dédié à Artémis, nous trouvons une prière semblable (III, 136 sq.) : "Artémis, que celui qui est mon ami sincère appartienne aux gens bienheureux qui ont ta faveur; que j'en sois moimême, ô reine, et que les chants soient toujours mon souci». Les motifs réitérés, nous le voyons, sont donc l'amitié avec la divinité et les chants, les hymnes qui doivent célébrer cette divinité.

Exception faite des vers finaux, où Théocrite expose sa réflexion personnelle, l'idylle n'offre pas de difficultés spéciales. Mais ce passage, surtout en ce qui concerne le vers 29 , est rempli d'obscurités. La variation des solutions proposées en donne la preuve : des plus drastiques, comme celle de Meineke et Hiller, extirpant tout simplement le vers, aux plus compliquées, comme celle de Vollgraff. Heureusement pour nous, Gow 6 dans son magnifique commentaire de l'œuvre de Théocrite offre un état précis et complet de la question, ce qui nous permet, à présent, de simplifier et de donner seulement les lignes générales sans entrer dans les détails.

Le passage présente, bien entendu, d'autres difficultés, mais nous nous bornerons ici à l'interprétation du vers 29 , celui qui offre plus de problèmes, et à son intégration dans le contexte.

Pour mieux saisir les différentes questions impliquées, nous avons reproduit quatre vers : 27-30. Les deux premiers hexamètres correspondent à l'édition de Gow. Ensuite, pour les vers 29-30, on a le texte du Parisinus 2726 du XVe siècle, $D$, pratiquement notre seul codex pour cette

6 A.S.F. Gow, Theocritus, II, Cambridge, 1950, p. 480 sq. 
idylle ${ }^{7}$ et celui du Papyrus d'Antinoé du Ve-VIe siècle ap. J.-C. qui dans ce vers diffère essentiellement du texte du manuscrit.

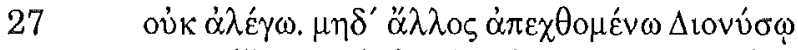
$\varphi \rho o v \tau i \zeta o \imath, \mu \eta \delta^{\prime} \varepsilon i \chi \alpha \lambda \varepsilon \pi \omega ́ \tau \varepsilon \rho \alpha \tau \hat{\omega} v \delta \varepsilon \mu \mathrm{o} \eta^{\prime} \sigma \alpha \iota$

Parisinus 2726

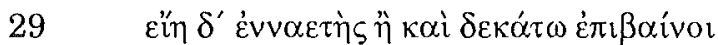

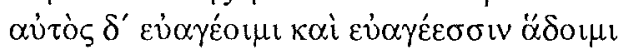

P. Antinoé

29

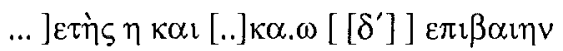

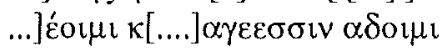

$\alpha \vee \tau(\imath \tau \circ v) \alpha[.] \ldots \varepsilon, \alpha$ $\alpha \delta \circ \iota \iota \varepsilon \iota \varsigma \theta \varepsilon \tau \eta$

L'hexamètre, en lui-même, n'est pas dépourvu de sens, mais une fois relié au contexte, sa signification est beaucoup moins satisfaisante. On peut le mettre en rapport avec la phrase précédente (c'est la lecture du

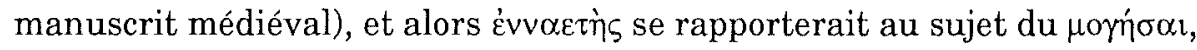
ou avec la phrase suivante (c'est ce que le papyrus fait; bien qu'il ait une fâcheuse lacune à l'endroit que devrait occuper eînv, cette lecture est

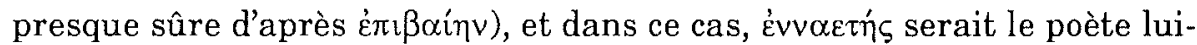
même. Mais dans les deux cas, les difficultés restent semblables.

Relié à ce qui précède, si ce que le poète a voulu mettre en relief c'est la jeunesse, et par conséquent, l'innocence de la victime, ce qui rendrait plus affreux le châtiment, on voit mal la progression 9-10. On aurait attendu, au contraire, un âge plus bas, pas un âge un peu plus haut. La

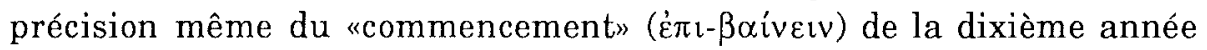
semble bizarre. Pourquoi pas «dix" ans tout simplement? D'ailleurs, qui est ce mystérieux petit, signalé avec tant de précision?

Wilamowitz, qui avait proposé un crime politique commis au sein d'une des familles hellénistiques régnantes ${ }^{8}$, voyait là le point faible de son argumentation : on ne pouvait pas apporter comme preuve le fait historique dont le poème serait le reflet.

On a essayé de résoudre ces difficultés en prétendant que ennaetes ne désigne pas exactement une jeune personne de neuf ans, mais serait le terme qui dénommerait un certain degré d'initiation mystérique, un myste de neuf ans d'ancienneté. Mais les problèmes au lieu de diminuer, s'aggravent. On doit alors supposer ${ }^{9}$ qu'il existait un tel degré initiatique

7 A.S.F. Gow, op. cit., I, p. LVI sq.

8 Op. cit., p. 213 sq.

9 Les preuves apportées ne sont pas satisfaisantes. Il est vrai que dans les Lois Sacrées de Cos (Inscr. Cos, 27-30 Paton-Hicks), relatives à la vente publique 
et qu'au début de la dixième année l'autorité du myste devenait plus grande au sein du thiasos, du groupe des initiés... Ces suppositions, cependant, ne clarifient pas non plus ce que le poète aurait prétendu dire avec ce vers, qui reste mystérieux et en outre très maladroit.

Le texte du papyrus offre la clef du problème. Le second hémistiche du vers 29 ne coïncide pas avec celui du manuscrit. Il ne s'agit pas seulement de la première ou de la troisième personne du verbe $\dot{\pi} \pi \_\alpha i ́ v \varepsilon \imath$. Dans

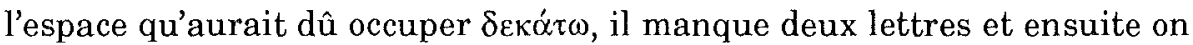
a $\kappa \alpha . \omega$. Le professeur Hunt, dans son commentaire à la fin du papyrus, précisait qu'on ne voyait pas clairement ce qu'était supposé être un tau, la lettre originale était obscurcie et une deuxième main, avec une encre plus sombre, avait refait le trait qui pouvait aussi bien s'interpréter comme un iota.

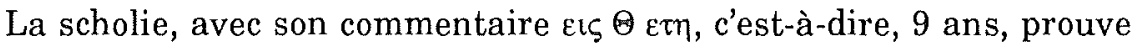
de façon indirecte qu'on ne lisait pas, sur le papyrus, $\delta \varepsilon \kappa \alpha ́ \tau \omega$. Sinon le scholiaste en aurait fait mention.

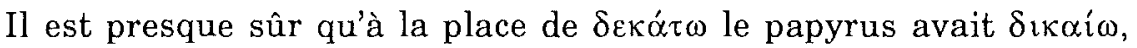

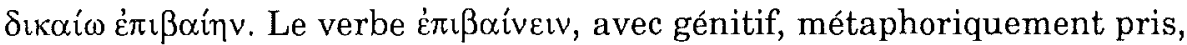
signifiant «arriver à», «atteindre» est parfaitement documenté en grec :

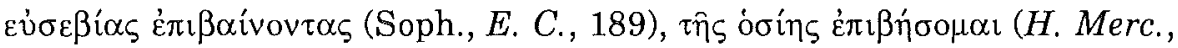

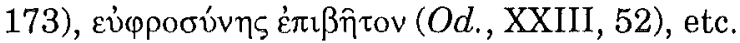

Mais en outre, et ceci est beaucoup plus déterminant, nous retrouvons la tournure dans le passage d'Hésiode qui évidemment inspira ces vers à Théocrite : Erga, 252 sq.

Le poète d'Ascra, dans les conseils à son frère Persès, loue «ceux qui jamais ne s'écartent de la justice", $\mu \grave{\eta} \pi \alpha \rho \varepsilon \kappa \beta \alpha i v o v \sigma \iota ~ \delta \iota \kappa \alpha i o v ~(226)$. Dans notre texte on a repris la même expression introduisant une petite variatio; c'est presque la même métaphore, mais pas tout à fait la même; c'est l'art allusif, tellement dans le goût alexandrin. Au lieu de

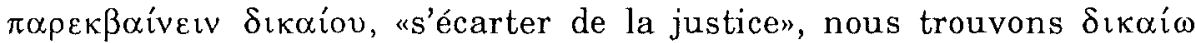

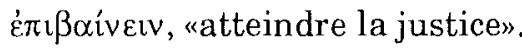

Nous avons ici une similitude frappante entre les deux passages. Une autre est oúk $\dot{\alpha} \lambda \varepsilon ́ \gamma o v \tau \varepsilon \zeta$ quelques vers plus bas (Erga, 251); et ce ne sont

de charges religieuses, on fait mention d'un âge minimum de 10 ans pour

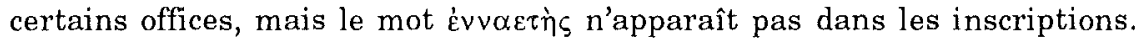
Toutes les explications proposées dans cette direction (Vollgraff, Blumenthal, Benedetto), sont trop compliquées et manquent d'une base réelle suffisamment documentée. Cf., p. ex., le résumé critique de Gow (op. cit., II, p. 481 sq.) de la "highly speculative solution" de Vollgraff. 
pas les seuls rapprochements que nous pourrions faire ${ }^{10}$. Mais, en plus de ces ressemblances ponctuelles, la réflexion de Théocrite, sa $\gamma v \dot{\omega} \mu \eta$, est exactement le contraire du vœu émis par Hésiode dans son désespoir (270):

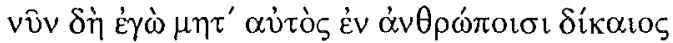

Eïn $\mu \eta \tau^{\prime} \dot{\varepsilon} \mu$ òs viòs.

«Puissé-je aujourd'hui cesser d'être juste, et moi et mon fils» est exactement la prière élevée par Théocrite mais à l'inverse : «Puissé-je atteindre la justice», "être juste». Il faut se souvenir des connotations que

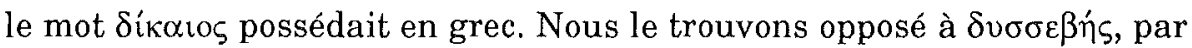

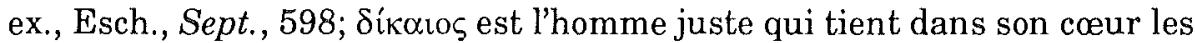
obligations envers les dieux, c'est un mot répété constamment dans les hymnes et dans le langage rituel; et dans ce passage de Théocrite nous le

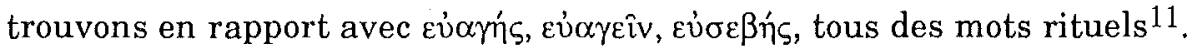

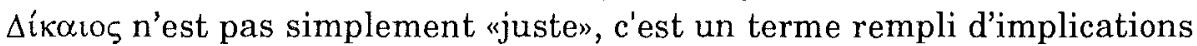
religieuses.

Comment est-il possible qu'un texte si facile et compréhensible ait subi une telle altération? Ce n'est pas une question de changement mécanique. Normalement, un texte se corrompt à partir d'une difficulté quelconque dans l'original que le copiste attentif cherche à résoudre. Dans notre passage il y avait un problème métrique, $\delta \imath \kappa \alpha i \omega$ avec la diphtongue scandée comme brève. Ces irrégularités métriques se produisent assez souvent quand on adapte une formule à un autre contexte ${ }^{12}$. Dans notre cas, il s'agit aussi d'une adaptation. On pouvait certainement trouver des exemples similaires avec la diphtongue raccourcie, par ex., $\pi \alpha \lambda \alpha$ เó $\varsigma$ dans

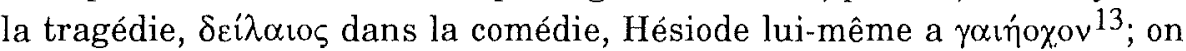
trouve aussi $\delta$ ík $\alpha$ lo, avec $\alpha \iota$ brève, dans Ménandre, dans un fragment orphique $^{14}$, mais d'habitude la diphtongue était longue et, dans notre cas, elle ne s'adaptait pas à sa place dans l'hexamètre. Alors, on a introduit

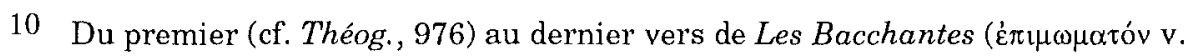
38 , hors d'ici seulement dans $E r g a, 13)$ l'influence hésiodique est évidente.

11 Cf. Gow, Theocritus, II, p. 483.

12 Cf. M.L. WEST dans son commentaire à Hésiode, Théog., 15.

13 Cf. M.L. West, loc, cit., et Greek Metre, Oxford, 1982, p. 11 sq., avec des références.

14 Epitr., 348 (cf. le commentaire ad loc. de A.W. Gomme et F.H. SandBaCh, Oxford, 1973) et Orph. Fragm., 247, 2 Kern. Cf. aussi la glose d'Hesychios,

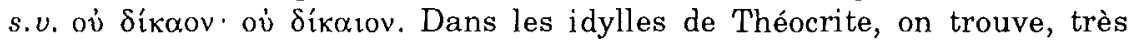

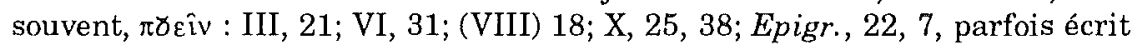

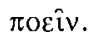


une petite modification, et $\delta \varepsilon \kappa \alpha \dot{\tau} \tau \omega$, en plus de sa similitude graphique,

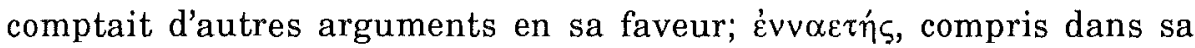
signification la plus courante "de neuf ans» entraînait fort naturellement la progression presque formulaire neuf-dix ${ }^{15}$, si fréquente depuis Homère, cette gradation étant encouragée d'autre part par $\dddot{\eta} \kappa \alpha i ́$. L'altération était donc facile.

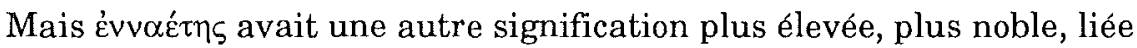

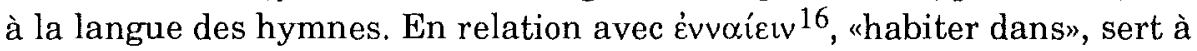
désigner les dieux qui vivent dans une région et qui, ainsi, la protègent

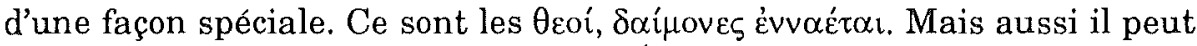
se rapporter aux habitants d'un pays. Évidemment, il existe une relation particulière entre les $\theta \varepsilon o i ̀ ~ c ́ v v \alpha \varepsilon ́ \tau \alpha l$ d'une région et ses habitants. De là, la récurrence du terme dans les hymnes, s'attachant au chœur des citoyens qui les entonnent. Ainsi, dans le péan d'Isyllos ${ }^{17}$, par exemple, nous

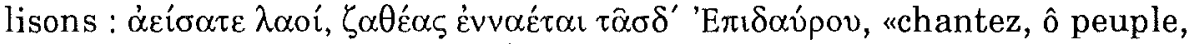
habitants de cette vénérable Épidaure». Et, en retour, ils attendent du

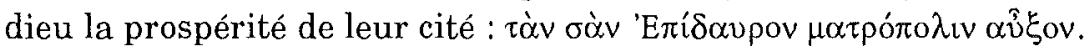

Philodème ${ }^{18}$ parle également de ces ennaetai dans son péan à

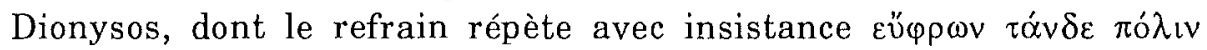
$\varphi u ́ \lambda \alpha \sigma \sigma^{\prime}$, "puisses-tu veiller, propice, sur cette ville !».

Et un fragment de Callimaque, récupéré dans un papyrus ${ }^{19}$, où il est

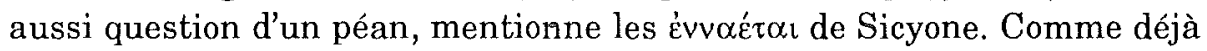
Wilamowitz le signalait ${ }^{20}$, le mot fait partie de formules de culte très anciennes. Les chœurs des habitants ${ }^{21}$, serviteurs dévoués de la divinité,

15 Les exemples de cette progression sont très nombreux. Cf. le commentaire de West à Hesiode, Théog., 636, et celui de G.S. KIRK à $I l$., II, 328 sq.

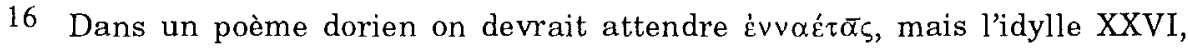
malgré le $\Delta \omega \rho i \delta \mathrm{l}$ de son titre, est très peu dorienne, aussi bien dans le

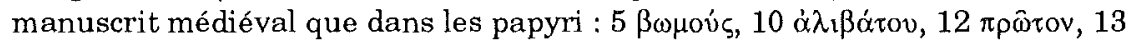

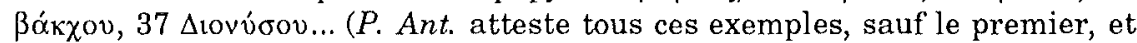
dans le petit fragment récupéré par E.G. TURNER, The Antinopolis, Papyri, III,

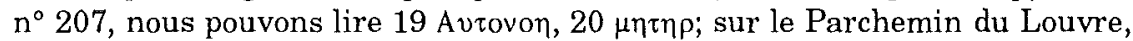
$21 \lambda \varepsilon \alpha i v] \eta$ s). Peut-être, avons-nous ici un des motifs de la corruption du

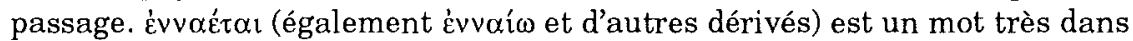
le goût alexandrin, cf. APOLL. RH., I, 921, 1126; II, 517, 1273; CALliM., fr. 186, $24 ; 177,36$, etc.

17 J.U. PowelL, Collectanea Alexandrina, Oxford, 1925, p. 132 sq.

18 Powell, op. cit., p. 165-171.

19 The Antinopolis Papyri, III, $\mathrm{n}^{\circ}$ 113. Cf. F. UEBEL, in AfP, 22 (1973), $\mathrm{n}^{\circ} 1220$.

20 Isyllos von Epidauros, Berlin, 1886, p. 14.

21 Puisque le sacerdoce était un service public, être citoyen était une condition indispensable pour arriver à toutes sortes de charges religieuses; on voit les 
qui ont à leur charge son culte, ses hymnes, sont plus redevables que nulle

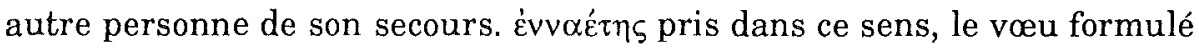
par le poète est : "puissé-je être ennaetes (appartenir au chœur des ennaetai)».

Nous avons donc deux hexamètres parallèles avec quatre optatifs désidératifs, deux dans chaque vers ${ }^{22}$. Trois d'entre eux échelonnés, le quatrièms, du point de vue logique, étant un développement du troisième (le poète aime bien ces symétries. On en trouve plusieurs exemples dans l'idylle, le filus notable peut-être au vers 18-19).

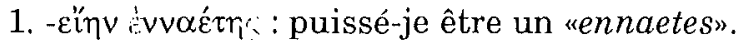

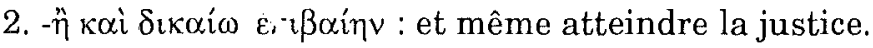

$\hat{\eta} \kappa \alpha i ́$ est une association de la conjonction disjonctive et de $\kappa \alpha i$ intensif exprimant une gradation.

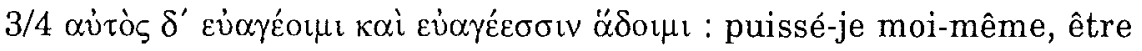
saint et plaire aux saints.

En ce qui concerne $\alpha$ ù òs $\delta^{\prime}$, tout d'abord, nous ne savons pas si le papyrus portait exactement ces mots. Av่ò $\delta^{\prime} \delta^{\prime}$ est la lecture du manuscrit, et il ne pouvait en être autrement, étant donné qu'il relie le vers 29 avec les précédents. La particule $\delta \varepsilon$ était nécessaire pour marquer l'opposition : la victime / pour ma part, je... Peut-être le papyrus avait une autre particule, par exemple $\tau \varepsilon, \alpha$ vं ós $\tau^{\prime}$.

De toute façon, $\alpha$ vós au vers 30 fait ressortir la personne du poète vis-à-vis de ces عن่ $\gamma^{\prime} \varepsilon \sigma \sigma ı v$ du deuxième hémistiche.

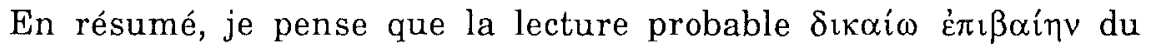
Papyrus d'Antinoé, qui pour les vers précédents aussi se montre plus digne de crédit que le manuscrit médiéval, est correcte, et le parallélisme évident de ces vers avec le passage d'Hésiode la confirme.

Le vers 29 s'intègre parfaitement dans l'ensemble avec la série des quatre optatifs renfermant les vœux du poète. La symétrie des quatre hémistiches s'accorde bien avec le style que nous trouvons dans le reste de

cités dresser soigneusement des listes de citoyens aptes pour ces fonctions, consignant non seulement le nom du père de chaque individu, mais aussi (par exemple à Cos), celui de la mère.

22 BLUMENTHAL, in RE, V A 2, c. 2020-2021 fut le premier à adopter la lecture du papyrus avec les quatre optatifs parallèles, mais en acceptant toujours la progression traditionnelle $9-10$ ans. Pourquoi, cependant, le poète aurait-il voulu avoir cet âge ? Ses arguments étaient peu convaincants. Cf. aussi V. DI BENEDETTO, in PP, 12 (1957), p. 271 sq. 
l'idylle, et les prières formulées sont dans la même ligne de celles de Callimaque que nous avons signalées.

Le passage a un sens satisfaisant, sans mystères ni obscurités. On ne doit rien présupposer : ni degrés d'initiation non confirmés, ni pauvres enfants massacrés, moins encore de festins cannibales ou une apologie de l'assassinat rituel.

Au fond, on se sent soulagé.

Departemento de Filología Clásica

Maria Teresa MOLINOS TEJADA Facultad de Filosofía y Letras

Universidad de Valladolid

E - 47002 VALLADOLID 